\title{
Fast wake-up time in obese patients: Which anesthetic is best?
}

\author{
Rita Katznelson, MD · Joseph A. Fisher, MD
}

Received: 5 March 2015/Accepted: 15 May 2015/Published online: 4 June 2015

(C) Canadian Anesthesiologists' Society 2015

Obesity has become an epidemic of global proportions with more than a third of the North American population being clinically overweight or obese. ${ }^{1}$ Anesthetizing obese patients can often be challenging as they have a higher incidence of comorbidities ${ }^{2}$ and an increased risk of a difficult airway. ${ }^{3}$ Furthermore, obese patients are prone to intraoperative pulmonary complications ${ }^{4}$ and acute upper airway obstruction and aspiration following tracheal extubation at the end of surgery. ${ }^{5}$ Obese patients have a higher incidence of sleep apnea and develop hypoxia during the early postoperative period. ${ }^{6}$ A faster recovery from anesthesia may decrease these risks by reducing the time patients require to resume control of their spontaneous breathing, restore airway protective reflexes, and regain efficient coughing.

Relationships between choice of anesthetic and recovery from anesthesia in obese patients remain unclear. Physiological changes related to obesity, such as an increase in cardiac output, may affect pharmacokinetics of anesthetic agents by, for example, increasing the volume of drug distribution and clearance; however, the extent of these changes is difficult to assess. Indeed, clinical trials have not shown significant clinical differences in the time to wake up between obese and nonobese patients anesthetized with inhalational agents. ${ }^{7}$ Furthermore, the fat solubility of anesthetics has not been shown to be important to anesthetic recovery times. ${ }^{8}$ All of the currently used inhalational anesthetics have a relatively high fat solubility. Thus, even in individuals with a normal body mass index (BMI), the capacitance of fat for anesthetics is generally

R. Katznelson, MD $(\bowtie) \cdot$ J. A. Fisher, MD

Department of Anesthesia and Pain Management, University

Health Network, University of Toronto, 200 Elizabeth Street,

EN3, Toronto, ON M5G 2C4, Canada

e-mail: rita.katznelson@uhn.ca too large to become saturated. Even after two to four hours of exposure at one minimal alveolar concentration (MAC), the saturation of isoflurane, sevoflurane, and desflurane in the fat is negligible relative to their MAC. The low partial pressure of anesthetic in fat, coupled with the relatively low blood flow to fatty tissues, results in little diffusion of the anesthetic back into blood during emergence; thus the anesthetic dissolved in the fat should contribute little to the prolongation of unconsciousness. ${ }^{8}$

The blood solubility of the anesthetic is likely more important in determining recovery time, as the blood is the second fastest compartment next to the lungs. ${ }^{8}$ The blood mediates the transfer of volatile anesthetic between the vesselrich group (VRG) and the lungs. The higher blood flow to capacitance ratio of the VRG compared with fat results in more rapid equilibration of anesthetic partial pressures with the blood. During emergence, the anesthetic exchange at the blood-lung interface becomes the most important factor determining the reduction of anesthetic concentration in the blood and thereby in the brain. ${ }^{8}$ Accordingly, an increase in minute ventilation and cardiac output accelerate the clearance of anesthetic from the blood. ${ }^{8}$ For a given minute ventilation, the anesthetic with the lowest blood solubility - i.e., desflurane - will exhibit the greatest clearance and the greatest reduction in blood partial pressure. Nevertheless, the differences in the blood-gas partition coefficient between desflurane (0.45) and those of sevoflurane and isoflurane (0.65 and 1.45, respectively) are quite small. The relative longer recovery time from sevoflurane and isoflurane compared with desflurane can be overcome by, for example, augmenting minute ventilation on emergence. ${ }^{9-11}$ It has been shown that as little as a two to threefold increase in minute ventilation, while avoiding hypocapnia (i.e., isocapnic hyperpnea), increases the rate of elimination of isoflurane compared with that of sevoflurane and desflurane in patients conventionally ventilated. . $^{9-1}$ 
In the current edition of the Journal, Liu et al. report a meta-analysis of recovery outcomes in obese patients who underwent general anesthesia. The authors analyzed 11 randomized controlled trials that included adult patients with a BMI $\geq 30 \mathrm{~kg} \cdot \mathrm{m}^{-2}$ who were anesthetized with desflurane, sevoflurane, isoflurane, or propofol for elective surgery. ${ }^{12}$ The primary outcomes were the time from terminating anesthetic administration to the patient's ability to respond to and follow simple commands, extubation, as well as the patient's ability to state their name or birthdate. Secondary outcomes included time to discharge from the postanesthesia care unit (PACU) and the incidence and severity of postoperative complications such as postoperative nausea and vomiting, pain, hypoxemia, and hemodynamic instability. The authors found that the patients anesthetized with desflurane were able to meet their primary arousal outcome endpoints 3.1$7.8 \mathrm{~min}$ faster than the patients anesthetized with comparator anesthetics. The study conclusion was that desflurane should be considered the inhaled anesthetic of choice in obese patients because of its more rapid and consistent early recovery profile.

The strength of the analysis is that the authors aggregated data from the most methodologically sound trials. In contrast, the main weakness comes from the small number of trials included and the variability in the measures of endpoints between studies. Of the 11 suitable studies, seven compared desflurane with sevoflurane, and two studies compared desflurane with isoflurane. The studies varied in their surgical procedures and anesthesia protocols, making the interpretation and generalization of the results more difficult. Furthermore, the review reported only emergence and early recovery characteristics. The incidence of adverse events, such as re-intubation, unexpected postoperative ventilation in the PACU, or clinically indicated admission to the intensive care unit would also have been relevant to the choice of anesthetic. The actual discharge time from the PACU may be a less reliable indicator of postanesthetic recovery than readiness for discharge.

Their analysis also included two studies comparing propofol-based anesthesia with desflurane, isoflurane, and sevoflurane, with only one trial using total intravenous anesthesia (TIVA). Accordingly, it is uncertain how to calculate equivalent doses of intravenous anesthetics in obese patients and how to gauge their clinical equivalence to the inhalation agents.

Obese patients show an increase in total body weight (TBW), lean body weight (LBW), and, to a greater extent, fat mass. This results in a lower ratio of LBW to TBW and a larger volume of distribution of lipophilic drugs. Obesity is also associated with an increase in cardiac output, which is proportionally related to LBW. It is still unclear how to calculate the bolus dose of the intravenous anesthetics. That is, should it be based on TBW to match the increase in volume of distribution or according to LBW to reflect the change in cardiac output? The next dilemma is how to compute the maintenance dose for the anesthetic intravenous infusion. A variety of pharmacokinetic models have proposed TBW or LBW-based calculations or plasma concentration and bispectral index parameters as a target. ${ }^{1}$

Different ways of calculating the doses of intravenous anesthetics can influence time to wake up in the immediate postoperative period and in the PACU. Until pharmacokinetic models for obese patients are standardized worldwide, it would be difficult to compare recovery parameters between TIVA and inhalation-based anesthesia in obese and non-obese patients.

We now return to the authors' conclusion that desflurane should be considered as the inhaled anesthetic of choice based on its more rapid recovery profile. First, when choosing an inhalational agent for an obese patient, there are more factors to consider than the speed of arousal. Obese patients frequently have comorbidities such as obstructive lung disease and hypertension. Isoflurane and sevoflurane may have advantages with respect to bronchodilation. In contrast, desflurane tends to increase airway resistance. ${ }^{13}$ Desflurane can also cause sympathetic stimulation and significantly elevated heart rate and blood pressure, particularly when its concentration is increased rapidly. ${ }^{14}$ Second, the recovery from general anesthesia is a complex process determined by many physiological and pharmacological factors that include context sensitive decrement time (duration of exposure, i.e., anesthetic concentration profile over time; whether the vaporizer is turned off from maintenance levels or tapered) ${ }^{15}$ and the presence of adjuvant anesthetics such as benzodiazepines, opiates, hypnotics, regional nerve blocks, and agents used for premedication. Compared with these factors and the minute ventilation at emergence, the independent effect of anesthetic blood solubility on arousal is quite small. ${ }^{9-11}$

By appropriately adapting other aspects of the conduct of the anesthesia, the anesthesiologist can tailor the anesthetic to the needs of the obese patient with any of the three common volatile agents so the patient may still be able to wake up equally rapidly in the operating room.

\section{Quel est le meilleur anesthésique pour favoriser un réveil rapide du patient obèse ?}

L'obésité est désormais une épidémie d'envergure mondiale: en effet, d'un point de vue clinique, plus d'un 
tiers des Nord-Américains sont considérés comme étant en surpoids ou obèses. ${ }^{1}$ Il est souvent difficile d'anesthésier les patients obèses étant donné qu'ils souffrent d'une incidence plus élevée de comorbidités ${ }^{2}$ et d'un risque accru de présenter des voies aériennes difficiles. ${ }^{3}$ De plus, les patients obèses sont souvent sujets à des complications pulmonaires peropératoires ${ }^{4}$ ainsi qu'à des problèmes d'obstruction des voies aériennes supérieures et d'aspiration après l'extubation trachéale en fin de chirurgie. ${ }^{5}$ Chez ces patients, on observe également une incidence plus élevée d'apnée du sommeil et ils sont souvent victimes d'hypoxie en début de période postopératoire. ${ }^{6}$ Une récupération plus rapide après l'anesthésie pourrait réduire ces risques en diminuant le temps requis par ces patients pour reprendre le contrôle de leur respiration spontanée, rétablir les réflexes protecteurs des voies aériennes et retrouver une toux efficace.

Les liens entre le choix de l'anesthésique et la récupération postanesthésique chez le patient obèse ne sont pas encore élucidés. Les changements physiologiques liés à l'obésité, notamment l'augmentation du débit cardiaque, pourraient avoir un impact sur la pharmacocinétique des agents anesthésiques : par exemple, ils pourraient augmenter le volume de distribution et de clairance d'un médicament. Il est toutefois difficile d'évaluer l'ampleur de ces changements. En effet, les études cliniques n'ont pas montré de différences significatives d'un point de vue clinique en matière de temps jusqu'au réveil entre les patients obèses et non obèses anesthésiés avec des agents volatils. ${ }^{7}$ De plus, il n'a pas été démontré que la solubilité lipidique des anesthésiques était importante en matière de temps de récupération après une anesthésie. ${ }^{8}$ Tous les agents volatils utilisés à l'heure actuelle ont une solubilité lipidique relativement élevée. Dès lors, même chez les personnes ayant un indice de masse corporelle (IMC) normal, la capacitance des tissus adipeux pour les agents anesthésiques est en général trop élevée pour atteindre la saturation. Même après deux à quatre heures d'exposition à une concentration alvéolaire minimale (MAC) de $1 \%$, la saturation de l'isoflurane, du sévoflurane et du desflurane dans les tissus adipeux est négligeable par rapport à leur MAC. La pression partielle basse des agents anesthésiques dans les tissus adipeux, couplée au débit sanguin relativement bas vers les tissus adipeux, n'entraîne qu'une faible distribution des agents anesthésiques dans le sang pendant le réveil; par conséquent, les agents anesthésiques dissous dans le tissus adipeux ne devraient avoir qu'un faible impact sur la prolongation de l'état inconscient. ${ }^{8}$

En revanche, la solubilité des agents anesthésiques dans le sang est probablement plus importante pour déterminer le temps de récupération, étant donné que le sang constitue le deuxième compartiment le plus rapide après les poumons. ${ }^{8}$ Le sang est le médiateur du transfert des agents anesthésiques volatils entre le groupe richement vascularisé (GRV) et les poumons. En raison du ratio plus élevé entre débit sanguin et capacitance du GRV par rapport aux tissus adipeux, l'équilibration des pressions partielles des agents anesthésiques avec le sang est plus rapide. Pendant le réveil, l'échange des agents anesthésiques qui se produit au niveau de l'interface sang-poumon devient le facteur le plus important pour déterminer la réduction de la concentration de l'anesthésique dans le sang et, par conséquent, dans le cerveau. ${ }^{8}$ Ainsi, une augmentation de la ventilation minute et du débit cardiaque accélère la clairance des agents anesthésiques du sang. ${ }^{8}$ Pour une ventilation minute donnée, l'anesthésique disposant de la solubilité sanguine la plus faible - soit le desflurane affichera la clairance la plus élevée et la plus importante réduction en pression partielle sanguine. Toutefois, les différences de coefficient de partage sang-gaz entre le desflurane $(0,45)$ et le sévoflurane et l'isoflurane $(0,65$ et 1,45 , respectivement) sont relativement faibles. Le temps de récupération relativement plus long qu'on observe avec le sévoflurane et l'isoflurane par rapport au desflurane peut être contré en augmentant la ventilation minute au réveil, par exemple. ${ }^{9-11}$ Il a été démontré qu'une augmentation de la ventilation minute d'aussi peu que deux ou trois fois peut, tout en évitant l'hypocapnie (c.-à-d. l'hyperpnée isocapnique), augmenter le taux d'élimination de l'isoflurane par rapport au sévoflurane et au desflurane chez les patients sous ventilation traditionnelle. ${ }^{9-11}$

Dans ce numéro du Journal, Liu et coll. rapportent une méta-analyse s'intéressant aux pronostics de récupération de patients obèses ayant subi une anesthésie générale. Les auteurs ont analysé 11 études randomisées contrôlées incluant des patients adultes avec un IMC $\geq 30 \mathrm{~kg} \cdot \mathrm{m}^{-2}$ et anesthésiés à l'aide de desflurane, de sévoflurane, d'isoflurane ou de propofol en vue d'une chirurgie non urgente. $^{12}$ Les critères d'évaluation principaux étaient l'intervalle entre la fin de l'administration de l'anesthésique et la capacité du patient à répondre et suivre des ordres simples, l'extubation, ainsi que la capacité du patient à déclarer son nom ou sa date de naissance. Les critères d'évaluation secondaires comprenaient le temps jusqu'au congé de la salle de réveil ainsi que l'incidence et la gravité des complications postopératoires telles que les nausées et vomissements postopératoires, la douleur, l'hypoxémie et l'instabilité hémodynamique. Les auteurs ont observé que les patients anesthésiés avec du desflurane étaient capables d'atteindre leurs critères d'évaluation à l'éveil 3,1-7,8 min plus rapidement que les patients anesthésiés à l'aide d'autres anesthésiques comparables. La conclusion de l'étude était qu'il faudrait considérer le desflurane comme l'agent anesthésique volatil de choix chez les patients obèses en raison de son profil de récupération plus rapide et constant. 
La force de l'analyse réside dans le fait que les auteurs ont agrégé les données des études les plus rigoureuses d'un point de vue méthodologique. En revanche, sa principale faiblesse vient du nombre restreint d'études incluses et de la variabilité des mesures de critères d'évaluation entre les études. Parmi les 11 études respectant les critères des auteurs, sept comparaient le desflurane au sévoflurane, et deux comparaient le desflurane à l'isoflurane. Les interventions chirurgicales et les protocoles d'anesthésie différaient d'une étude à une autre, ce qui rend l'interprétation et la généralisation des résultats plus ardues. De plus, la méta-analyse ne rapporte que les données de réveil et de récupération précoce. L'incidence d'événements néfastes tels que la réintubation, une ventilation postopératoire inattendue en salle de réveil ou une admission indiquée d'un point de vue clinique à l'unité des soins intensifs, aurait également joué un rôle dans le choix de l'anesthésique. Le moment réel de congé de la salle de réveil pourrait être un indicateur moins fiable de récupération postanesthésique que le fait d'être prêt à recevoir son congé.

Leur analyse a également inclus deux études comparant une anesthésie au propofol avec le desflurane, l'isoflurane, et le sévoflurane, et une seule étude utilisant une anesthésie intraveineuse totale (TIVA). Par conséquent, nous ne savons pas exactement comment calculer des doses équivalentes d'anesthésiques intraveineux chez les patients obèses, ni comment estimer leur équivalence clinique aux agents volatils.

Chez un patient obèse, on observe une augmentation du poids corporel réel (PCR), de la masse maigre (MM) et, dans une plus grande mesure, de la masse grasse. Cela entraîne un rapport plus bas entre la MM et le PCR et un volume plus important de distribution des médicaments lipophiles. L'obésité est également associée à une augmentation du débit cardiaque, qui est proportionnellement lié à la MM. À l'heure actuelle, nous ne savons pas comment calculer la dose de bolus des anesthésiques intraveineux. Autrement dit, devrait-elle être basée sur le PCR afin de correspondre à l'augmentation du volume de distribution ou sur la MM afin de refléter le changement de débit cardiaque? Un autre dilemme porte sur la façon de calculer la dose de maintien de la perfusion intraveineuse d'anesthésique. Divers modèles pharmacocinétiques ont proposé des calculs fondés sur le PCR ou sur la MM ou des paramètres de concentration plasmatique ou d'indice bispectral comme cible. $^{1}$

Différentes façons de calculer les doses d'anesthésiques intraveineux peuvent influencer le temps jusqu'au réveil en période postopératoire immédiate et en salle de réveil. Tant que les modèles pharmacocinétiques destinés aux patients obèses ne sont pas standardisés au niveau mondial, il reste difficile de comparer les paramètres de récupération entre une TIVA et une anesthésie par inhalation - que le patient soit obèse ou non.

Retournons maintenant à la conclusion des auteurs, selon laquelle il faudrait envisager le desflurane comme l'anesthésique volatil de choix en raison de son profil de récupération plus rapide. Tout d'abord, lorsqu'on choisit un agent volatil pour prendre en charge un patient obèse, il y a plusieurs autres facteurs dont il faut tenir compte outre la rapidité de réveil. Les patients obèses présentent souvent des comorbidités telles qu'une pneumopathie obstructive ou de l'hypertension. L'isoflurane et le sévoflurane pourraient avoir des avantages en ce qui touche à la bronchodilatation. En revanche, le desflurane a tendance à augmenter la résistance des voies aériennes. ${ }^{13}$ Le desflurane peut également provoquer une stimulation sympathique et une fréquence cardiaque et une pression artérielle significativement élevées, particulièrement lorsqu'on augmente rapidement sa concentration. ${ }^{14}$ Deuxièmement, la récupération suite à une anesthésie générale est un processus complexe influencé par de nombreux facteurs physiologiques et pharmacologiques, notamment un temps de décroissance intimement lié au contexte (durée d'exposition, c.-à-d. profil de concentration anesthésique au fil du temps; si le vaporisateur est éteint lorsqu'on a atteint les niveaux de maintien ou s'il a été progressivement baissé) ${ }^{15}$ et la présence d'anesthésiques adjuvants tels que les benzodiazépines, les opiacés, les agents hypnotiques, les blocs nerveux régionaux, et les agents utilisés en prémédication. Par rapport à ces facteurs et la ventilation minute au réveil, l'effet indépendant de la solubilité dans le sang de l'agent anesthésique sur le réveil est relativement faible. ${ }^{9-11}$

En adaptant de façon appropriée d'autres aspects de l'anesthésie, l'anesthésiologiste peut personnaliser l'anesthésie selon les besoins du patient obèse avec n'importe lequel des trois agents volatils facilement accessibles de façon à ce que le patient puisse se réveiller tout aussi rapidement en salle d'opération.

Conflicts of interest Rita Katznelson and Joseph A. Fisher are a part of the team that developed ClearMate ${ }^{\mathrm{TM}}$ and are shareholders in Thornhill Research Inc, partially owned by the University Health Network.

Conflit d'intérêt Rita Katznelson et Joseph A. Fisher font partie de l'équipe qui a mis au point ClearMate ${ }^{\mathrm{TM}}$ et sont actionnaires de Thornhill Research Inc., qui appartient en partie au réseau University Health Network.

\section{References}

1. Ingrande J, Lemmens $H J$. Anesthetic pharmacology and the morbidly obese patient. Curr Anesthesiol Rep 2013; 3: 10-7. 
2. Reeder BA, Senthilselvan A, Despres JP, et al. The association of cardiovascular disease risk factors with abdominal obesity in Canada. Canadian Heart Health Surveys Research Group. CMAJ 1997; 157(Suppl 1): S39-45.

3. Hood DD, Dewan DM. Anesthetic and obstetric outcome in morbidly obese parturients. Anesthesiology 1993; 79: 1210-8.

4. Eichenberger A, Proietti S, Wicky S, et al. Morbid obesity and postoperative pulmonary atelectasis: an underestimated problem. Anesth Analg 2002; 95: 1788-92.

5. Murphy C, Wong DT. Airway management and oxygenation in obese patients. Can J Anesth 2013; 60: 929-45.

6. Ahmad S, Nagle A, McCarthy RJ, Fitzgerald PC, Sullivan JT, Prystowsky $J$. Postoperative hypoxemia in morbidly obese patients with and without obstructive sleep apnea undergoing laparoscopic bariatric surgery. Anesth Analg 2008; 107: 138-43.

7. Lemmens HJ, Saidman LJ, Eger EI 2nd, Laster MJ. Obesity modestly affects inhaled anesthetic kinetics in humans. Anesth Analg 2008; 107: 1864-70.

8. Eger EI 2nd, Saidman LJ. Illustrations of inhaled anesthetic uptake, including intertissue diffusion to and from fat. Anesth Analg 2005; 100: 1020-33.

9. Katznelson R, Minkovich L, Friedman Z, Fedorko L, Beattie WS, Fisher JA. Accelerated recovery from sevoflurane anesthesia with isocapnic hyperpnoea. Anesth Analg 2008; 106: 486-91.
10. Katznelson $R$, Naughton $F$, Friedman $Z$, et al. Increased lung clearance of isoflurane shortens emergence in obesity: a prospective randomized-controlled trial. Acta Anaesthesiol Scand 2011; 55: 995-1001.

11. Sakata DJ, Gopalakrishnan NA, Orr JA, White JL, Westenskow $D R$. Hypercapnic hyperventilation shortens emergence time from isoflurane anesthesia. Anesth Analg 2007; 104: 587-91.

12. Liu FL, Cherng YG, Chen SY, et al. Postoperative recovery after anesthesia in morbidly obese patients: a systematic review and meta-analysis of randomized controlled trials. Can J Anesth 2015; 62: this issue. DOI: 10.1007/s12630-015-0405-0.

13. Dikmen Y, Eminoglu E, Salihoglu Z, Demiroluk S. Pulmonary mechanics during isoflurane, sevoflurane and desflurane anaesthesia. Anaesthesia 2003; 58: 745-8.

14. Weiskopf RB, Moore MA, Eger EI 2nd, et al. Rapid increase in desflurane concentration is associated with greater transient cardiovascular stimulation than with rapid increase in isoflurane concentration in humans. Anesthesiology 1994; 80: 1035-45.

15. Eger EI 2nd, Shafer SL. Tutorial: context-sensitive decrement times for inhaled anesthetics. Anesth Analg 2005; 101: 688-96. 